Proceedings

Juntendo Medical Journal

2016. 62 (Suppl 1), 70-74

\title{
MR Imaging for Sportology; Non-Invasive Visualization of the Brain and Muscles
}

\author{
SHIGEKI AOKI*1), MASAAKI HORI*1), ATSUSHI NAKANISHI*1), ChrisTINA ANDICA*1), \\ MiSAKI NAKAZAWA*1), KANAKO SATO*1), KOUICHI ASAHI*1) 2), KeIGO SHIMOJI*1) 3) \\ *1) Department of Radiology, Juntendo University Graduate School of Medicine, Tokyo, Japan, *2) Juntendo University \\ Shizuoka Hospital, Shizuoka, Japan, *3) Department of Diagnostic Radiology, Tokyo Metropolitan Geriatric Hospital, Tokyo, \\ Japan
}

\begin{abstract}
Recent advances of MR imaging allow us to visualize the functional and structural changes of the brain not only in the patients with neurological disorders, but also in normal subjects. Sophisticated MR techniques, such as resting-state functional MR and diffusion MR imaging, can depict subtle brain changes in normal subjects before metabolic syndrome as well as brain changes after a few weeks muscle training. Minimal structural changes in the brain after repeated minor head trauma during sports are now highlighted, because advanced MR techniques can be used to show substantial changes in the brain before neurological decline.

By diffusion tensor imaging (DTI), we can explore microstructure of the tissue in vivo through analysis of water diffusion direction and restriction. DTI was developed mainly in the central nervous system to visualize the white matter tracts and their networks. Plasticity of the brain white matter has been reconfirmed with this technique. Skeletal muscles also have the direction and restriction of water diffusion and can be analyzed by DTI as well.

Recent advances of MR techniques for sportology, especially diffusion MR imaging, will be presented in this paper.
\end{abstract}

Key words: sportology, diffusion tensor imaging, MR imaging

\section{Introduction}

Recent advances of MR imaging (MRI) allow us to evaluate subtle changes of the human brain in vivo. Functional MRI can demonstrate local activity changes related to the "task" in the brain. Tasks can be auditory, visual, motor or other complicated procedures. Changes or differences of regional brain volume can be evaluated by the voxel-based morphometry using three-dimensional T1weighted images of MRI. MR spectroscopy represents a spectrum of several brain metabolites semi-quantitatively. Diffusion tensor imaging (DTI) or diffusion MR analysis can uniquely evaluate the white matter tracts through precise analysis of water diffusion. There are several methods to analyze brain using DTI such as tract-specific analysis (TSA), tract-based special statistics (TBSS), and connectome. In this paper, we would like to present the usefulness of MR imaging for sportology, especially about DTI.

\section{Quick review of diffusion tensor imaging of the brain}

The white matter could not explored precisely, because it was difficult to track the fibers using specimen. DTI is a unique technique to visualize the white matter tract. DTI has been proposed by Basser in $1994^{1)}$ and developed rapidly in these years. DTI is a model that used to analyze the diffusion of water molecule in vivo, assuming the form of diffusion in one voxel as an ellipsoid (Figure-1). In the brain, diffusion of water molecules is anisotropic due to white matter fibers. Diffusion parallel to the white matter fibers is larger

Corresponding author: Shigeki Aoki

Department of Radiology, Juntendo University Graduate School of Medicine

2-1-1 Hongo, Bunkyo-ku, Tokyo 113-8421, Japan

TEL: +81-3-3813-3111 E-mail: saoki@juntendo.ac.jp

The $2{ }^{\text {nd }}$ Congress, International Academy of Sportology〔Held on Sep. 12, 2015〕

〔Received Dec. 18, 2015〕 


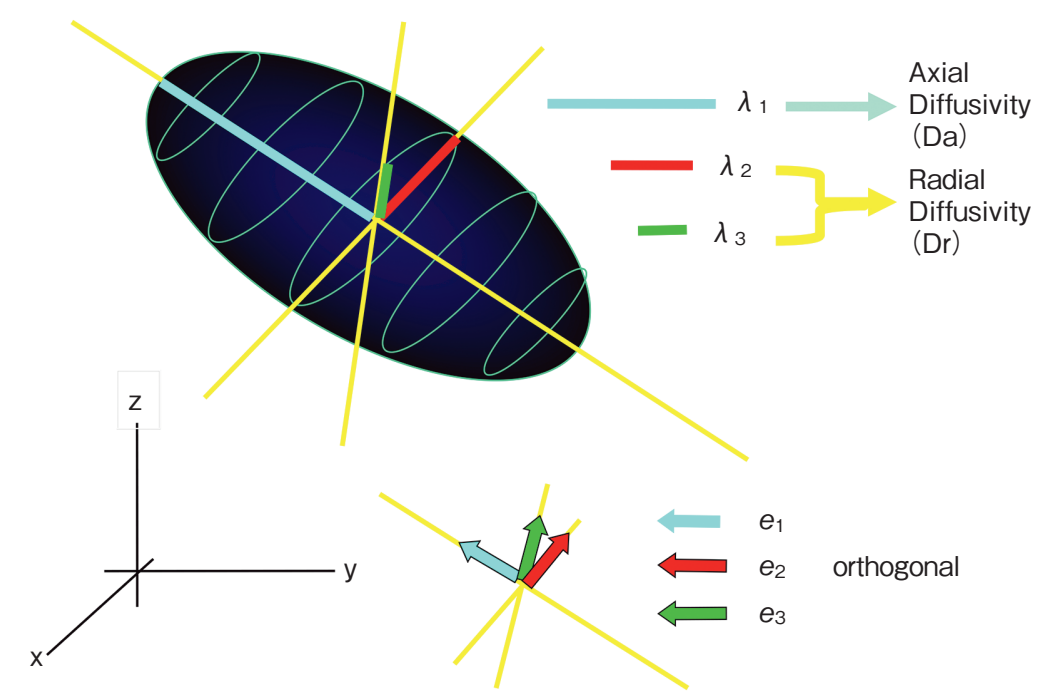

Figure-1 Diffusion tensor ellipsoid and Lambda 1, 2 and 3

than the one perpendicular to the fibers. The component of diffusion parallel to the white matter is Lambda $1\left(\lambda_{1}\right)$ or axial diffusivity (Da). The component of diffusion perpendicular to the white matter is Lambda $2\left(\lambda_{2}\right)$ and $3\left(\lambda_{3}\right)$ (Lambda 2 and 3 are perpendicular to each other). Average of Lambda 1 and 2 is called radial diffusivity (Dr). The standardized standard deviation of Lambda 1, 2, and 3 is Fractional Anisotropy (FA). When the white matter fibers are densely packed, water molecules move mainly along (parallel to) the fibers, this is due to the myelin and other membranes. The FA value becomes high when the Lambda 1 is large, but Lambda 2 and 3 are small. Decreased FA of the white matter fibers indicates deterioration or degeneration of white matter fibers.

By tracking or chasing the Lambda 1 direction three dimensionally, we can visualize the white matter tracts ${ }^{2)}$ 3) (Figure-2, 3). The tracking algorism so called FACT (Fiber Assignment by Continuous Tracking) has been proposed by Mori ${ }^{2)}$. Dense and thick bundles such as the corticospinal tracts, the cingulate, uncinate and fornix are clearly visualized (Figure-3). The tracts without crossing fibers are clearly visualized by DTI. Region of interest (ROI) analysis $^{4)}$ and TSA $^{5)}$ 6) are developed to quantify the tract integrity measuring parameters within the tract.

Whole brain statistical analysis can be implicated in DTI data. The statistical parametric map (SPM) is widely used for functional MRI analysis and can also be used for DTI analysis. TBSS (tract based special statistics) ${ }^{7)}$ is a newly developed technique to analyze DTI and other diffusion MR imaging. Using whole brain statistical analysis, we can explore regional brain abnormalities without the hypothesis of locations (Figure-4).

Non-Gaussian diffusion analysis is now highlighted to analyze precise microstructures using multi-shell (v-value) multi-MPG (>30) MR acquisition $^{8)}$.

\section{Plasticity of brain by training}

Changes of the brain after training or exercise that seen by brain MRI had been reported in several conditions. Aerobic exercise training increases brain volume in aging humans ${ }^{9)}$. Volume changes of both gray and white matter, especially on the frontal lobe was observed. Changes of volume in one year had been reported (VOSS HBM). Significant increase of Fractional Anisotropy (FA) of DTI also had been observed.

Plasticity of the brain has been presented by using DTI after training of three-ball juggling ${ }^{10)}$. After 6 week hard training of juggling, increase of whole brain FA, especially at the right parietal lobe, probably related to special recognition, was observed. In addition, after 4 week rest, this increased FA was decreased.

Early plasticity of the brain after learning can be evaluated as well ${ }^{11)}$. Changes of DTI parameters of 

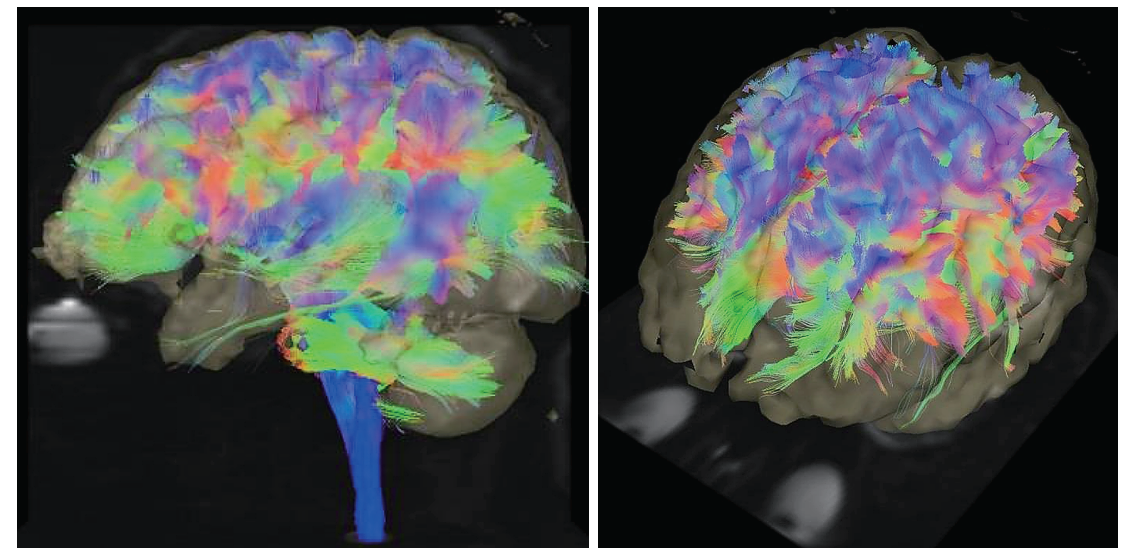

Figure-2 Diffusion tensor tractography of the whole brain

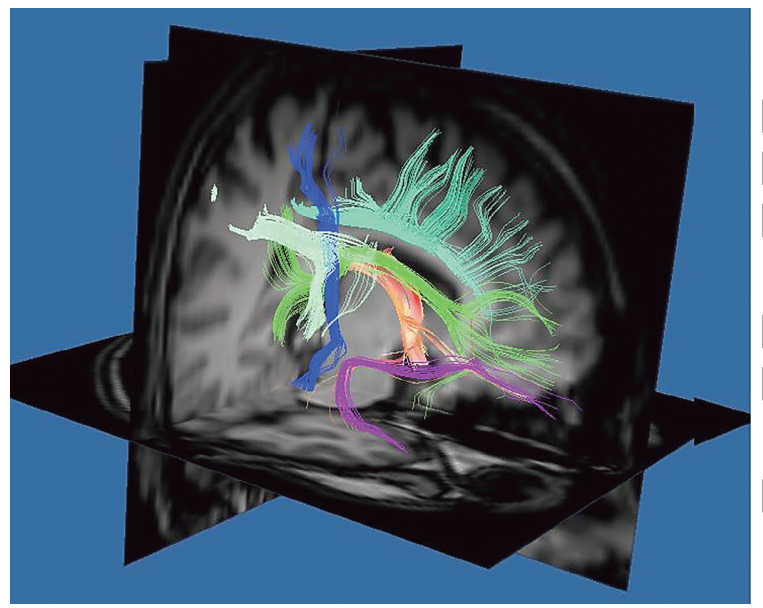

Cingululm

CST

Sup fronto-

occipital

fasciculus

$\square$ Uncinate

$\square$ Superior

longitudinal

sasciculus

$\square$ Fornix

Figure-3 The limbic system and corticospinal tracts are the tracts that can be visualized consistently by diffusion tensor tractography

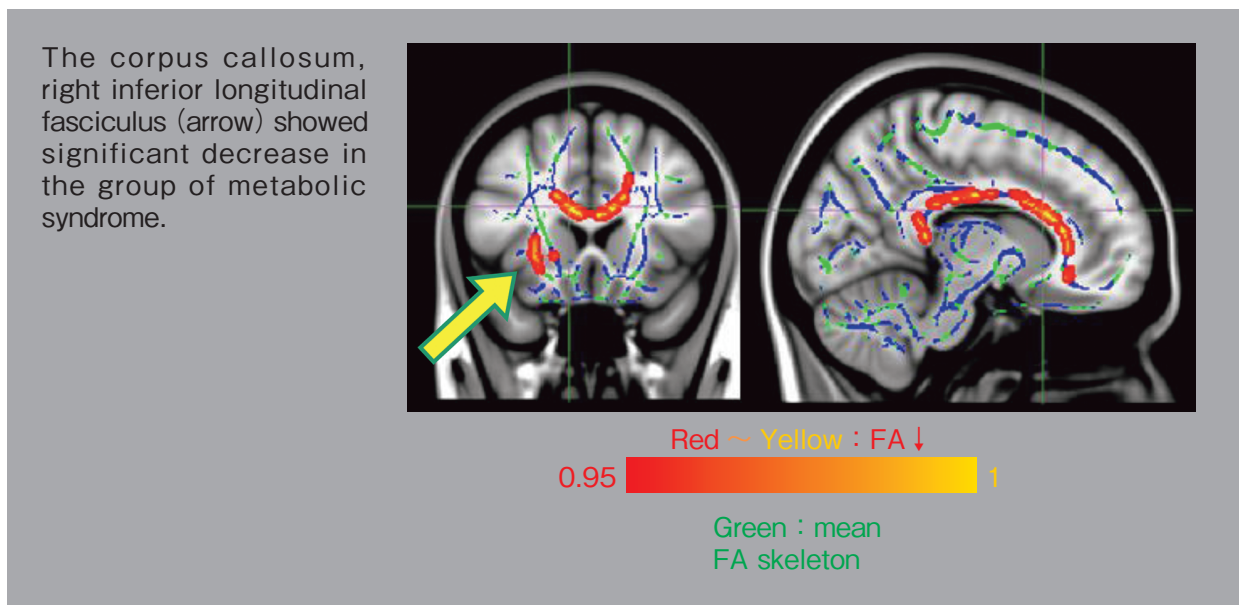

Figure-4 Brain changes in matabolic syndrome: TBSS analysis. The corpus callosum and external capsule showed significant deterioration. The external capsule was actually the inferior longitudinal fasciculus.

(Shimoji K, et al: Diabetes Care, 2013; 36: 696-700 ${ }^{13)}$ ) 
limbic system (hippocampus and para-hippocampus) were observed after spatial learning and memory task.

\section{Diffusion tensor imaging of metabolic syndrome}

Changes of the brain in individuals with metabolic syndrome have been explored by TBSS ${ }^{122}{ }^{13)}$ (Figure-4). TBSS revealed a significant decrease of FA in the corpus callosum and the right longitudinal fasciculus. TSA of the right longitudinal fasciculus showed significant correlation between FA of the tract and body-mass index. These changes of the long tract might be related to faint deterioration, possibly related to metabolic syndrome.

\section{Diffusion tensor imaging and minor trauma}

Subtle changes of the minor brain trauma of athletes such as in soccer and American football have been highlighted. National football league in the United States and General Electric had a big project called Head Health Challenges. This project focuses on the development of technologies that can detect early stage mild traumatic brain injuries. Many papers about minor head trauma or concussion have been published recently. It is because of the development of new imaging methods, especially DTI.

Diffusion tensor imaging has been used to evaluate minor trauma due to sports. Subtle changes of repeated heading during soccer have been reported. The quantity of heading prior 12 month and DTI of 37 amateur soccer players were evaluated. "FA at three locations in the temporo-occipital white matter is lower, with a threshold that varied according to location (885-1550 headings per year) $(\mathrm{p}<.00001)$. Lower levels of FA were also associated with poorer memory scores $(p<.00001)$, with a threshold of 1800 headings per year." ${ }^{14)}$ Changes of DTI metrics of the individual subjects after sports-related concussion has been reported ${ }^{15)}$.

\section{DTI outside the brain}

Diffusion tensor imaging can be used to visualize the complexity of the muscle structures. The bundles of the muscles can be clearly demonstrated using diffusion tensor tractography. Change of apparent diffusion coefficient has been observed even in the Trunk muscles between before and after training ${ }^{16)}$.

Change of diffusion tensor parameters after long-distance running (marathon) also has been reported ${ }^{17)}$. Marathon runners were scanned before, 2 days after and 3 weeks after marathon. Change of DTI parameters was observed in different muscles in different degrees at the level of upper leg ${ }^{17)}$.

\section{References}

1) Basser PJ, Mattiello J, LeBihan D: Estimation of the effective self-diffusion tensor from the NMR spin echo. J Magn Reson B, 1994; 103: 247-254.

2) Mori S, Crain BJ, Chacko VP, van Zijl PC: Three-dimensional tracking of axonal projections in the brain by magnetic resonance imaging. Ann Neurol, 1999; 45: 265-269.

3) Masutani Y, Aoki S, Abe O, Hayashi N, Otomo K: MR diffusion tensor imaging: recent advance and new techniques for diffusion tensor visualization. Eur J Radiol, 2003; 46: 53-66.

4) Abe O, Aoki S, Hayashi N, et al: Normal aging in the central nervous system: quantitative MR diffusion-tensor analysis. Neurobiol Aging, 2002; 23: 433-441.

5) Kunimatsu A, Aoki S, Masutani Y, Abe O, Mori H, Ohtomo K: Three-dimensional white matter tractography by diffusion tensor imaging in ischaemic stroke involving the corticospinal tract. Neuroradiology, 2003; 45: 532-535.

6) Aoki S, Iwata NK, Masutani Y, et al: Quantitative evaluation of the pyramidal tract segmented by diffusion tensor tractography: feasibility study in patients with amyotrophic lateral sclerosis. Radiat Med, 2005; 23: 195-199.

7) Smith SM, Jenkinson M, Johansen-Berg H, et al: Tract-based spatial statistics: voxelwise analysis of multi-subject diffusion data. Neuroimage, 2006; 31: 1487-1505.

8) Hori M, Fukunaga I, Masutani Y, et al: Visualizing non-Gaussian diffusion: clinical application of q-space imaging and diffusional kurtosis imaging of the brain and spine. Magn Reson Med Sci, 2012; 11: 221-233.

9) Colcombe SJ, Erickson KI, Scalf PE, et al: Aerobic exercise training increases brain volume in aging humans. J Gerontol A Biol Sci Med Sci, 2006; 61: 1166-1170.

10) Scholz J, Klein MC, Behrens TE, Johansen-Berg H: Training induces changes in white-matter architecture. 
Nat Neurosci, 2009; 12: 1370-1371.

11) Sagi Y, Tavor I, Hofstetter S, Tzur-Moryosef $S$, Blumenfeld-Katzir T, Assaf Y: Learning in the fast lane: new insights into neuroplasticity. Neuron, 2012; 73: 1195-1203.

12) Shimoji K, Uka T, Tamura $Y$, et al: Diffusional kurtosis imaging analysis in patients with hypertension. Jpn J Radiol, 2014; 32: 98-104.

13) Shimoji K, Abe O, Uka T, et al: White matter alteration in metabolic syndrome: diffusion tensor analysis. Diabetes Care, 2013; 36: 696-700.

14) Lipton ML, Kim N, Zimmerman ME, et al: Soccer heading is associated with white matter microstructural and cognitive abnormalities. Radiology, 2013; 268: 850-857.

15) Bazarian JJ, Zhu T, Blyth B, Borrino A, Zhong J: Subject-specific changes in brain white matter on diffusion tensor imaging after sports-related concussion. Magn Reson Imaging, 2012; 30: 171-180.

16) Yanagisawa O, Matsunaga N, Okubo Y, Kaneoka K: Noninvasive evaluation of trunk muscle recruitment after trunk exercises using diffusion-weighted MR Imaging. Magn Reson Med Sci, 2015; 14: 173-181.

17) Froeling M, Oudeman J, Strijkers GJ, et al: Muscle changes detected with diffusion-tensor imaging after long-distance running. Radiology, 2015; 274: 548-562. 